\title{
A MINIMUM PROBLEM FOR THE EPSTEIN ZETA-FUNCTION
}

\author{
by R. A. RANKIN
}

(Received 28th January, 1953)

1. In some recent work by D. G. Kendall and the author $\dagger$ on the number of points of a lattice which lie in a random circle the mean value of the variance emerged as a constant multiple of the value of the Epstein zeta-function $Z(s)$ associated with the lattice, taken at the point $s=\frac{3}{2}$. Because of the connexion with the problems of closest packing and covering it seemed likely that the minimum value of $Z\left(\frac{3}{2}\right)$ would be attained for the hexagonal lattice ; it is the purpose of this paper to prove this and to extend the result to other real values of the variable $s$.

Let

$$
h(m, n)=\alpha m^{2}+2 \delta m n+\beta n^{2}
$$

be a positive definite quadratic form of determinant $\alpha \beta-\delta^{2}$ equal to unity. In particular, the special forms $Q(m, n)$ and $q(m, n)$ are defined as follows :

$$
Q(m, n)=\frac{2}{3} \sqrt{ } 3 q(m, n)=\frac{2}{3} \sqrt{ } 3\left(m^{2}+m n+n^{2}\right) .
$$

We consider the Epstein zeta-function $\ddagger$.

$$
Z_{h}(s)=\sum_{m=-\infty}^{\infty} \sum_{n=-\infty}^{\infty}\{h(m, n)\}^{-s},
$$

where the dash denotes, as always, the exclusion of the term $m=n=0$. This double series is absolutely convergent for $\Re s>1$. The function $Z_{h}(s)$ can be continued over the whole s-plane and is regular except for a simple pole of residue 1 at $s=1$.

It is easily shown that in the particular case $h(m, n)=Q(m, n)$

$$
Z_{Q}(s)=6\left(\frac{1}{2} \sqrt{ } 3\right)^{s} \zeta(s) L(s)
$$

where $\zeta(s)$ is the Riemann zeta-function and $L(s)$ is the Dirichlet $L$-series

$$
L(s)=1-\frac{1}{2^{s}}+\frac{1}{4^{s}}-\frac{1}{5^{s}}+\frac{1}{7^{s}}-\frac{1}{8^{s}}+\ldots .
$$

We shall, throughout the paper, be concerned with real values of $s$ in the half-plane of convergence. We prove the

Theorem. For all $s \geqslant 1.035, Z_{h}(s) \geqslant Z_{Q}(s)$. Equality occurs only when $h$ and $Q$ are equivalent forms.

In view of the closeness of the lower bound 1.035 to unity and the fact that all the functions $Z_{h}(s)$ are asymptotically equal as $s \rightarrow 1+0$ it seems very likely that the conclusion of the theorem remains valid for all $s>1$, but I have been unable to prove this. Further, I have not been able to find any single method which is applicable to the whole range $s \geqslant 1 \cdot 035$, as will be seen from the proof.

\footnotetext{
$\dagger$ " On the number of points of a given lattice in a random hypersphere." (To appear in the Quarterly Journal.)

$\ddagger$ For the general theory of $Z_{h}(s)$ see Max Deuring, "Zetafunktionen quadratischer Formen" J. reine angew. Math. 172 (1935), 226-252. 
2. In this section we introduce some new notation and prove 10 lemmas. In the first place, as is well known, we may, by means of a unimodular transformation on the variables $m$ and $n$, assume that $\alpha$ is the minimum of the form for all pairs of integers $m, n$, not both zero, and that

$$
0 \leqslant 2 \delta \leqslant \alpha \leqslant \beta, \quad 1 \leqslant \alpha \beta \leqslant \frac{4}{3} .
$$

With these restrictions on the coefficients, the Theorem states that, for $s \geqslant 1 \cdot 035, Z_{h}(s) \geqslant Z_{Q}(s)$ and that equality occurs only for $h=Q$.

We now introduce some symmetry by defining

so that

$$
\gamma=\alpha+\beta-2 \delta=\alpha+\beta-2 \sqrt{ }(\alpha \beta-1),
$$

and, by (5),

$$
2(\beta \gamma+\gamma \alpha+\alpha \beta)-\left(\alpha^{2}+\beta^{2}+\gamma^{2}\right)=4
$$

Also define

$$
0<\alpha \leqslant \beta \leqslant \gamma \leqslant \alpha+\beta \text {. }
$$

$$
\left.\begin{array}{l}
f(m, n)=\beta m^{2}+(\beta+\gamma-\alpha) m n+\gamma n^{2}=h(-n, m+n) \\
g(m, n)=\gamma m^{2}+(\gamma+\alpha-\beta) m n+\alpha n^{2}=h(m+n,-m)
\end{array}\right\} .
$$

Then we find that

$$
2(g h+h f+f g)-\left(f^{2}+g^{2}+h^{2}\right)=4 q^{2},
$$

and deduce that

$$
f=g+h \pm 2 \sqrt{ }\left(g h-q^{2}\right), \quad g=h+f \pm 2 \sqrt{ }\left(h f-q^{2}\right), \quad h=f+g \pm 2 \sqrt{ }\left(f g-q^{2}\right) .
$$

LEMMA 1. If $\alpha+\beta+\gamma \leqslant 4$, the minus sign must be taken in each of the three relations (11). Proof. We prove that $f+g-h$ cannot take negative values. We have

$$
f+g-h=(\beta+\gamma-\alpha) m^{2}+(3 \gamma-\alpha-\beta) m n+(\gamma+\alpha-\beta) n^{2} .
$$

By (8), the coefficients of $m^{2}$ and $n^{2}$ are non-negative and the determinant of the form is, by (7),

$$
(\beta+\gamma-\alpha)(\gamma+\alpha-\beta)-\frac{1}{4}(3 \gamma-\alpha-\beta)^{2}=4-\frac{1}{4}(\alpha+\beta+\gamma)^{2} \geqslant 0
$$

In a similar way it can be shown that $g+h-f$ and $h+f-g$ are not negative.

We now introduce new variables $x, y$ and $z$ defined by

so that we have

$$
x=\delta / \alpha, \quad y=1 / \alpha, \quad z=x+i y,
$$

$$
h(m, n)=\frac{|m+n z|^{2}}{y},
$$

and the conditions (8) imply that $z$ belongs to the modular region $\mathbb{Z}$ defined by

Put

$$
0 \leqslant x \leqslant \frac{1}{2}, \quad y>0, \quad x^{2}+y^{2} \geqslant 1 \text {. }
$$

$$
\phi(x, y ; s)=y^{s}\left\{1+\frac{1}{\left(x^{2}+y^{2}\right)^{s}}+\frac{1}{\left\{(x-1)^{2}+y^{2}\right\}^{s}}\right\}=\frac{1}{\alpha^{s}}+\frac{1}{\beta^{s}}+\frac{1}{\gamma^{s}} .
$$

We prove

LEMMa 2. If $s>2$ we have

$$
\frac{1}{\alpha^{s}}+\frac{1}{\beta^{s}}+\frac{1}{\gamma^{s}} \geqslant 3\left(\frac{1}{2} \sqrt{ } 3\right)^{s} .
$$

Equality holds only when $\alpha=\beta=\gamma=2 / \sqrt{ } 3$. 
Proof. By Hölder's inequality, since $s>2$,

$$
\phi(x, y ; 2) \leqslant\{\phi(x, y ; s)\}^{2} s 3^{1-2 / s},
$$

and equality can only occur when $x=\frac{1}{2}, y=\frac{1}{2} \sqrt{ } 3$. Accordingly, it is enough to prove that $\phi(x, y ; 2) \geqslant \frac{9}{4}$ in $\mathbb{Z}$ and that equality occurs only for $x=\frac{1}{2}, y=\frac{1}{2} \sqrt{ } 3$ and $x=0, y=1$.

Write $y^{2}=\eta$ and put

$$
\psi(x, \eta)=\phi(x, y ; 2)=\eta\left\{1+\frac{1}{\left(x^{2}+\eta\right)^{2}}+\frac{1}{\left\{(1-x)^{2}+\eta\right\}^{2}}\right\}
$$

Then

and is positive if

$$
\frac{\partial \psi}{\partial x}=-4 \eta\left[\frac{x}{\left(x^{2}+\eta\right)^{3}}-\frac{1-x}{\left\{(1-x)^{2}+\eta\right\}^{3}}\right]
$$

Since $\eta \geqslant 1-x^{2}$ in $\frac{\partial \psi}{\partial x}>0$ if

$$
\frac{x}{1-x}<\left\{\frac{x^{2}+\eta}{(1-x)^{2}+\eta}\right\}^{3}
$$

i.e. if $0 \leqslant x<\frac{1}{4}(3-\sqrt{ } 5)=x_{0}$, say.

$$
\frac{x}{1-x}<\frac{1}{8(1-x)^{3}}
$$

Suppose now that $x \geqslant x_{0}$. We have

and

$$
\frac{\partial \psi}{\partial \eta}=1+\frac{x^{2}-\eta}{\left(x^{2}+\eta\right)^{3}}+\frac{(1-x)^{2}-\eta}{\left\{(1-x)^{2}+\eta\right\}^{3}},
$$

$$
\frac{1}{2} \frac{\partial^{2} \psi}{\partial \eta^{2}}=\frac{\eta-2 x^{2}}{\left(x^{2}+\eta\right)^{4}}+\frac{\eta-2(1-x)^{2}}{\left\{(1-x)^{2}+\eta\right\}^{4}}
$$

Now, by evaluating the derivatives at $\eta=a+b$ it is easily verified that

$$
(\eta-2 a)(\eta+b)^{4}+(\eta-2 b)(\eta+a)^{4}
$$

is not negative for $\eta \geqslant a+b$, and since $y^{2} \geqslant x^{2}+(1-x)^{2}$ in 迎 we deduce that $\partial^{2} \psi / \partial \eta^{2} \geqslant 0$ in $\mathbb{1}$. Hence, since $\eta \geqslant 1-x^{2}$ in 㚼,

since $x_{0} \leqslant x \leqslant \frac{1}{2}$.

$$
\frac{1}{2 y} \frac{\partial \phi}{\partial y}=\frac{\partial \psi}{\partial \eta} \geqslant 2 x^{2}-\frac{x}{4(1-x)^{2}} \geqslant 0
$$

Accordingly we deduce that $\psi(x, \eta)$ attains its minimum value in either on the line $x=0,\left\{y \geqslant 1\right.$ or on the $\operatorname{arc} x^{2}+y^{2}=1,0 \leqslant x \leqslant \frac{1}{2}$. Now, if $x=0, y \geqslant 1$ we have

$$
\frac{\partial \psi}{\partial \eta}=\frac{\eta-1}{\eta^{2}(\eta+1)^{3}}\left\{(\eta+1)^{4}-\eta^{2}\right\} \geqslant 0
$$

so that $\phi(0, y ; 2) \geqslant \phi(0,1 ; 2)=\frac{9}{4}$.

Finally, if $x^{2}+y^{2}=1,0 \leqslant x \leqslant \frac{1}{2}$ we put

so that $1 \leqslant v \leqslant 3$, and obtain

$$
x=\frac{v-1}{v+1}, \quad y^{2}=\frac{4 v}{(v+1)^{2}}
$$

$$
\phi(x, y ; 2)=\frac{1}{4} v+\frac{8 v}{(v+1)^{2}}=\phi(v)
$$


say. Since

$$
\phi^{\prime}(v)=\frac{(v-3)\left(v^{2}+6 v-11\right)}{4(v+1)^{3}}
$$

we-deduce that as $v$ increases from 1 to $3, \phi(v)$ increases from $\frac{\theta}{4}$ to a maximum at $v=2 \sqrt{ } 5-3$ and then decreases to a minimum value of $\frac{9}{4}$ at $v=3$. This completes the proof of Lemma 2 .

We note that all that has been assumed in the proof of Lemma 2 is that $8>\mathbf{2}$ and that $\alpha, \beta$ and $\gamma$ satisfy (7) and the inequalities (8). We use this fact to prove

LEMma 3. If $\alpha+\beta+\gamma \leqslant 4$ and $s>2$ then, for any values of $m$ and $n$, not both zero,

$$
\frac{1}{\{f(m, n)\}^{s}}+\frac{1}{\{g(m, n)\}^{s}}+\frac{1}{\{h(m, n)\}^{s}} \geqslant 3\left(\frac{\sqrt{ } 3}{2 q}\right)^{s} .
$$

Equality occurs only when $\alpha=\beta=\gamma=2 / \sqrt{ } 3$.

Proof. Denote by $a, b$ and $c$ the three quantities $f / q, g / q, h / q$ arranged in ascending order of magnitude. Then, by (10) and Lemma 1,

and

$$
2(b c+c a+a b)-\left(a^{2}+b^{2}+c^{2}\right)=4,
$$

so that, by Lemma 2 ,

$$
0<a \leqslant b \leqslant c \leqslant a+b \text {, }
$$

$$
a^{-s}+b^{-s}+c^{-s} \geqslant 3\left(\frac{1}{2} \sqrt{ } 3\right)^{s} \text {. }
$$

Equality occurs only when $f=g=h=2 q / \sqrt{ } 3$, and then, since $f+g+h=(\alpha+\beta+\gamma) q$, we have $\alpha+\beta+\gamma=2 \sqrt{ } 3$. I.e., in terms of $x$ and $y,\left(x-\frac{1}{2}\right)^{2}+\left(y-\frac{1}{2} \sqrt{ } 3\right)^{2}=0$, so that $x=\frac{1}{2}, y=\frac{1}{2} \sqrt{ } 3$ and therefore $\alpha=\beta=\gamma=2 / \sqrt{ } 3$.

LEMMA 4. If $\alpha+\beta+\gamma \geqslant 4$ and $s>2$, then

$$
\alpha^{-8}+\beta^{-8}+\gamma^{-8} \geqslant 2+2^{-s} \text {. }
$$

Proof. In terms of the point $z$, defined by (12), we have to consider the region $0 \leqslant x \leqslant \frac{1}{2}$, $\left(x-\frac{1}{2}\right)^{2}+(y-1)^{2} \geqslant \frac{1}{4}$, which we denote by $\mathbf{\text { Tit*. }}$

Write

$$
r_{1}=\left(x^{2}+y^{2}\right)^{\frac{1}{2}}, \quad r_{2}=\left\{(x-1)^{2}+y^{2}\right\}^{\frac{1}{2}},
$$

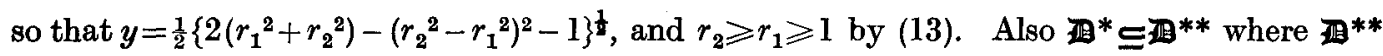
is the part of 7 in which $r_{2} \geqslant \sqrt{ } 2$. Then

$$
\phi(x, y ; s)=2^{-s}\left\{2\left(r_{1}^{2}+r_{2}^{2}\right)-\left(r_{2}^{2}-r_{1}^{2}\right)^{2}-1\right\}^{\frac{1}{s} s}\left(1+r_{1}^{-2 s}+r_{2}{ }^{-2 s}\right),
$$

and it is easily verified that, for $r_{2}$ constant,

where

$$
\frac{\partial \phi}{\partial r_{1}}=2^{1-s} s r_{1}\left\{2\left(r_{1}^{2}+r_{2}^{2}\right)-\left(r_{2}^{2}-r_{1}^{2}\right)^{2}-1\right\}^{\frac{1}{2} s-1} \omega
$$

Now

$$
\omega=\left(r_{2}^{2}-r_{1}^{2}\right)\left(r_{2}^{2}-2\right) r_{1}^{-2 s-2}+\left(1+r_{2}^{2}-r_{1}^{2}\right)\left(1+r_{2}^{-2 s}\right)-3 r_{1}^{-2 s}+r_{1}^{-2 s-2} \text {. }
$$

$$
\frac{1}{2 r_{2}} \frac{\partial}{\partial r_{2}}\left(1+r_{2}{ }^{2}-r_{1}{ }^{2}\right)\left(1+r_{2}{ }^{-2 s}\right)=r_{2}{ }^{-2 s-2}\left\{\left[r_{2}{ }^{2 s}-(s-1)\right]{r_{2}}^{2}+s\left(r_{1}{ }^{2}-1\right)\right\}>0,
$$

since $r_{2}^{2} \geqslant 2, r_{1}^{2} \geqslant 1$ and $2^{s}>s-1$. Thus, since $r_{2} \geqslant r_{1}$ in 双** we have

and therefore

$$
\left(1+r_{2}^{2}-r_{1}^{2}\right)\left(i+r_{2}^{-2 s}\right) \geqslant 1+r_{1}^{-2 s}
$$

$$
\omega \geqslant 1+r_{1}^{-2 s}-3 r_{1}^{-2 s}+r_{1}^{-2 s-2} \geqslant 1-2 r_{1}^{-2 s}+r_{1}^{-4 s} \geqslant 0 \text {. }
$$


Hence $\partial \phi / \partial r_{1} \geqslant 0$ in $\mathbb{R}^{* *}$ and it follows that $\phi(x, y ; s)$ attains its minimum in $\mathbb{1}^{*}$ on the line $x=0, y \geqslant 1$. Since

we have

$$
\begin{aligned}
\frac{\partial}{\partial y} \phi(0, y ; s) & =s y^{-s-1}\left(y^{2}-1\right)\left\{\frac{y^{2 s}-1}{y^{2}-1}-\frac{y^{2 s}}{\left(y^{2}+1\right)^{s+1}}\right\} \\
& \geqslant s y^{-s-1}\left(y^{2}-1\right)(s-1) \geqslant 0
\end{aligned}
$$

$$
\phi(0, y ; s) \geqslant \phi(0,1 ; s)=2+2^{-s},
$$

which completes the proof of the Lemma.

Lemma 5. The real function $f(t)$ is defined for all real $t$ and possesses the following properties : (i) $f(t), f^{\prime}(t)$ and $f^{\prime \prime}(t)$ are continuous for all $t$, (ii) the integrals $\int_{-\infty}^{\infty} f(t) d t$ and $\int_{-\infty}^{\infty}\left|f^{\prime \prime}(t)\right| d t$ converge, (iii) $f(t)$ and $f^{\prime}(t)$ tend to zero as $t \rightarrow \pm \infty$, (iv) $f^{\prime \prime}(t)$ is negative for $t_{1}<t<t_{2}$, but otherwise non-negative. Then $S=\sum_{n=-\infty}^{\infty} f(n)$ is convergent and, for some real $\vartheta$ satisfying $|\vartheta| \leqslant 1$,

$$
S=\int_{-\infty}^{\infty} f(t) d t+\frac{1}{8} \vartheta\left\{f^{\prime}\left(t_{1}\right)-f^{\prime}\left(t_{2}\right)\right\}
$$

Proof. We use the Euler-Maclaurin sum-formula in the form :

$$
\sum_{n=-M}^{N} f(n)=\int_{-M}^{N} f(t) d t+\frac{1}{2}\{f(N)+f(-M)\}+\int_{-M}^{N} r_{1}(t) f^{\prime \prime}(t) d t
$$

where $r(t)=t-[t]-\frac{1}{2}$ and

$$
r_{1}(t)=-\int_{0}^{t} r(u) d u
$$

It is easily shown that $0 \leqslant r_{1}(t) \leqslant \frac{1}{8}$ for all $t$, and we deduce that the infinite series converges and that

$$
S-\int_{-\infty}^{\infty} f(t) d t=\int_{-\infty}^{\infty} r_{1}(t) f^{\prime \prime}(t) d t=R
$$

say. From condition (iv) it follows that

$$
\frac{1}{8}\left\{f^{\prime}\left(t_{2}\right)-f^{\prime}\left(t_{1}\right)\right\}=\frac{1}{8} \int_{t_{1}}^{t_{2}} f^{\prime \prime}(t) d t \leqslant R \leqslant \frac{1}{8}\left(\int_{-\infty}^{t_{1}}+\int_{t_{2}}^{\infty}\right) f^{\prime \prime}(t) d t=\frac{1}{8}\left\{f^{\prime}\left(t_{1}\right)-f^{\prime}\left(t_{2}\right)\right\},
$$

which completes the proof.

In the next two lemmas we are concerned with the function $Z_{h}(s)$ expressed in terms of the variables $x$ and $y$, and write

$$
G(x, y)=Z_{h}(s)=\sum_{m} \sum_{n}^{\prime} y^{s}|m z+n|^{-2 s}
$$

Lemma 6.† For $s>1$,

where

$$
\frac{1}{2 s} G_{y}(x, y)=y^{s-1} \zeta(2 s)-y^{-s}(s-1) \zeta(2 s-1) \frac{\Gamma\left(\frac{1}{2}\right) \Gamma\left(s-\frac{1}{2}\right)}{\Gamma(s+1)}+R^{\prime}
$$

$$
\left|R^{\prime}\right| \leqslant \frac{1}{2} y^{-s-2} \zeta(2 s+1)\left\{s \frac{(2 s+1)^{s+\frac{1}{2}}}{(2 s+2)^{s+1}}+2(s+1) \frac{(2 s+3)^{s+\frac{s}{2}}}{(2 s+4)^{s+2}}\right\} .
$$

$\dagger$ Deuring (loc. cit.) gives a somewhat similar formula for the function $G(x, y)$, but with a different for $m$ of remainder and without the explicit numerical constants which are essential for our purpose. 
Proof. We have, because of uniform convergence,

$$
\begin{aligned}
\frac{1}{2 s} G_{y}(x, y) & =\frac{1}{2 s} \sum_{m=-\infty} \sum_{n=-\infty}^{\Sigma^{\prime}} \frac{\partial}{\partial y}\left\{\frac{y}{(m x+n)^{2}+m^{2} y^{2}}\right\}^{s} \\
& =\frac{1}{2} \sum_{m} \sum_{n}^{\prime} y^{s-1} \frac{|m z+n|^{2}-2 m^{2} y^{2}}{|m z+n|^{2 s+2}} \\
& =y^{s-1} \zeta(2 s)-\sum_{m=1}^{\infty}\left\{\sum_{n=-\infty}^{\infty} \frac{2 m^{2} y^{s+1}}{|m z+n|^{2 s+2}}-\sum_{n=-\infty}^{\infty} \frac{y^{s-1}}{|m z+n|^{2 s}}\right\}
\end{aligned}
$$

We now apply Lemma 5 to each of the two inner sums. Put

where $\rho=s$ or $s+1$. Then

$$
f(t)=\frac{y^{\rho}}{|m z+t|^{2 \rho}},
$$

$$
f^{\prime}(t)=-\frac{2 \rho y^{\rho}(m x+t)}{|m z+t|^{2 \rho+2}}, \quad f^{\prime \prime}(t)=\frac{2 \rho y^{\rho}\left\{(2 \rho+1)(m x+t)^{2}-m^{2} y^{2}\right\}}{|m z+t|^{2 \rho+4}},
$$

so that the conditions are satisfied with

We deduce that

$$
m x+t_{1}=-\frac{m y}{\sqrt{ }(2 \rho+1)}, \quad m x+t_{2}=\frac{m y}{\sqrt{ }(2 \rho+1)} .
$$

$$
\sum_{-\infty}^{\infty} f(n)=\int_{-\infty}^{\infty} \frac{y^{\rho} d t}{m z+\left.t\right|^{2 \rho}}+\frac{\vartheta \rho}{2 m^{2 \rho+1} y^{\rho+1}} \frac{(2 \rho+1)^{\rho+1}}{(2 \rho+2)^{\rho+1}},
$$

and, since the integral is

$$
\frac{y^{1-\rho}}{m^{2 \rho-1}} \frac{\Gamma\left(\frac{1}{2}\right) \Gamma\left(\rho-\frac{1}{2}\right)}{\Gamma(\rho)},
$$

the result follows from (16) on taking $\rho=s+1$ and $s$ in the two parts of the sum over $n$.

Lemma 7. If $s>1$ and $y \geqslant \frac{3}{2}$ then $G_{y}(x, y)>0$.

Proof. By Lemma 6,

where

$$
\frac{1}{2 s} G_{y}(x, y) \geqslant y^{s-1} \zeta(2 s)-y^{-s} \phi_{1}(s)-y^{s-2} \phi_{2}(s),
$$

and

$$
\phi_{1}(s)=\frac{\Gamma\left(\frac{1}{2}\right) \Gamma\left(s+\frac{1}{2}\right)}{\Gamma(s+1)} \cdot \frac{2(s-1) \zeta(2 s-1)}{2 s-1},
$$

say. Since

$$
\phi_{2}(s)=y^{-2 s} \zeta(2 s+1)\left\{\frac{1}{2} s \frac{(2 s+1)^{s+\frac{1}{2}}}{(2 s+2)^{s+1}}+(s+1) \frac{(2 s+3)^{s+\frac{3}{2}}}{(2 s+4)^{s+2}}\right\}=\phi_{3}(s)+\phi_{4}(s),
$$

and

$$
\zeta(2 s-1) \leqslant 1+\int_{1}^{\infty} u^{1-2 s} d u=\frac{2 s-1}{2(s-1)},
$$

$$
\frac{\Gamma\left(\frac{1}{2}\right) \Gamma\left(s+\frac{1}{2}\right)}{\Gamma(s+1)}=\int_{-\infty}^{\infty} \frac{d t}{\left(t^{2}+1\right)^{s+1}}<\frac{\Gamma\left(\frac{1}{2}\right) \Gamma\left(\frac{3}{2}\right)}{\Gamma(2)}=\frac{1}{2} \pi,
$$

it follows that $\phi_{1}(s)<\frac{1}{2} \pi$.

Also, it is easily proved by differentiation that both $\phi_{3}(s)$ and $\phi_{4}(s)$ are decreasing functions of $s$ for $y \geqslant \frac{3}{2}$. Thus we have, since $\phi_{2}(1) \leqslant 0 \cdot 8174 y^{-2}$,

$$
\frac{1}{2 s} G_{y}(x, y) \geqslant y^{s-1}\left\{\zeta(2 s)-\frac{1}{2} \pi y^{1-2 s}-0 \cdot 8174 y^{-3}\right\}=y^{s-1} \phi_{9}(s, y),
$$


say. In order to show that $\phi_{3}(s, y)>0$ for $y \geqslant \frac{3}{2}$ it is enough to prove that $\phi_{3}\left(s, \frac{3}{2}\right)>0$. Now, for $1 \leqslant s \leqslant 1 \cdot 3$ we have

$$
\phi_{3}\left(s, \frac{3}{2}\right) \geqslant \zeta(2 \cdot 6)-\frac{1}{2} \pi\left(\frac{3}{2}\right)^{-1}-0 \cdot 8174\left(\frac{3}{2}\right)^{-3}>0.016>0,
$$

for $1 \cdot 3 \leqslant s \leqslant 2$ we have

$$
\phi_{3}\left(s, \frac{3}{2}\right) \geqslant \zeta(4)-\frac{1}{2} \pi\left(\frac{3}{2}\right)^{-1 \cdot 6}-0 \cdot 8174\left(\frac{3}{2}\right)^{-3}>0 \cdot 019>0,
$$

and for $s \geqslant 2$ we have

This completes the proof.

$$
\phi_{3}\left(s, \frac{3}{2}\right) \geqslant 1-\left(\frac{1}{2} \pi+0 \cdot 8174\right)\left(\frac{3}{2}\right)^{-3}>0 \cdot 292>0 .
$$

LEMMA 8. Let $h$ and $\mu$ be fixed positive numbers and suppose that the function $H(u)$ possesses continuous derivatives $H^{\prime}(u), H^{\prime \prime}(u)$ for $u \geqslant u_{0}$ and that $H^{\prime \prime}(u)>\mu^{2} H(u)$ for all $u>u_{0}$. Then

for all $u \geqslant u_{0}$.

$$
H_{1}(u) \equiv H(u+2 h)-2 \cosh \mu h H(u+h)+H(u)>0,
$$

Proof. This follows from the formula

$$
H_{1}(u)=e^{-\mu u} \int_{u}^{u+h} e^{2 \mu v} d v \int_{v}^{v+h} e^{-\mu w}\left\{H^{\prime \prime}(w)-\mu^{2} H(w)\right\} d w
$$

which is easily checked by integration.

For the remainder of the paper we write

$$
H(u)=u^{s+\frac{1}{2}} K_{s-\frac{1}{1}}(u), \cdots
$$

where $K_{s-\frac{1}{2}}(u)$ is a Bessel function.

Lemma 9. If $0 \leqslant \mu<1$ then $H^{\prime \prime}(u)>\mu^{2} H(u)$ provided either that (i) $u\left(1-\mu^{2}\right) \geqslant 2 s$ where $s \geqslant 1$ or (ii) $\left(1-\mu^{2}\right) u \geqslant 3\left(1-\mu^{2}\right)+2 s \mu^{2}$, when $1 \leqslant s \leqslant 2$.

Proof. By using the relations (1) and (5) of $\S 3.71$ of G. N. Watson's Bessel Functions, $\dagger$ we obtain

and since

$$
H^{\prime \prime}(u)-\mu^{2} H(u)=\left(1-\mu^{2}\right) u^{s+\frac{3}{2}} K_{s-\frac{1}{2}}(u)-2 s u^{s-\frac{1}{2}} K_{8-\frac{3}{2}}(u),
$$

$$
K_{\nu}(u)=\int_{0}^{\infty} e^{-u \cosh t} \cosh \nu t d t
$$

and $\cosh \left(s-\frac{1}{2}\right) t \geqslant \cosh \left(s-\frac{3}{2}\right) t$, the first part of the Lemma follows.

Suppose now that $1 \leqslant s \leqslant 2$. Then if we substitute for $K_{s-\frac{1}{2}}(u)$ in terms of $K_{s-\frac{3}{2}}(u)$ and $K_{s-\frac{s}{2}}(u)$ in (18) and use the fact that $K_{\nu}(z)=K_{-\nu}(z)$, we get

$$
H^{\prime \prime}(u)-\mu^{2} H(u)=\left(1-\mu^{2}\right) u^{s+\frac{1}{2}} K_{\frac{s}{2}-s}(u)-\left\{3\left(1-\mu^{2}\right)+2 s \mu^{2}\right\} u^{s-\frac{1}{2}} K_{\frac{3}{2}-s}(u),
$$

and (ii) follows from this in a similar manner.

Lemma 10. We have $H^{\prime \prime}(u)>\mu^{2} H(u)$ for all $u \geqslant u_{0}$ in the following cases : (i) $u_{0}=\frac{6}{5} \pi r$, $\mu=\mu_{r}=\frac{r}{u} \cosh ^{-1}\left(1+2^{1-2 s}\right) \quad(1 \leqslant s \leqslant 2 ; \quad r=1,2), \quad$ (ii) $\quad u_{0}=\frac{6}{5} \pi r, \quad \mu=\mu_{r}=\frac{r}{u} \cosh ^{-1}\{\zeta(2 s-1)\}$ $(1 \cdot 035 \leqslant s \leqslant 2 ; r=3,4,5, \ldots)$, (iii) $u_{0}=2 \pi, \mu=\frac{1}{u} \cosh ^{-1}\{\zeta(2 s-1)\}(2 \leqslant s \leqslant 3)$.

Proof. (i) Put $a=\frac{6}{5} \pi$. We suppose first that $1 \leqslant s \leqslant \frac{3}{2}$. By Lemma 9 (i), it suffices to show that

$$
a-\frac{1}{a}\left\{\cosh ^{-1}\left(1+2^{1-2 s}\right)\right\}^{2} \geqslant 2 s,
$$

since $u\left(1-\mu_{r}{ }^{2}\right)$ is an increasing function of $u$. This is true since

$$
\begin{aligned}
\cosh \{a(a-2 s)\}^{\frac{1}{2}} \geqslant & \cosh \{a(a-3)\}^{\frac{1}{2}}>2 \cdot 838>\frac{3}{2} \geqslant 1+2^{1-2 s} . \\
& + \text { Cambridge, } 1922 .
\end{aligned}
$$


Suppose next that $\frac{3}{2} \leqslant s \leqslant 2$. Since $\left(1-\mu_{r}^{2}\right)(u-3)-2 s \mu_{r}^{2}$ increases with $u$, it is enough, by Lemma 9 (ii), to show that

i.e. that

$$
a^{2}(a-3)-(a-3+2 s)\left\{\cosh ^{-1}\left(1+2^{1-2 s}\right)\right\}^{2} \geqslant 0,
$$

$$
1+2^{1-2 s} \leqslant \cosh \left\{a\left(\frac{a-3}{a-3+2 s}\right)^{\frac{1}{2}}\right\} \text {. }
$$

This follows since $1+2^{1-2 s} \leqslant \frac{5}{4}<2 \cdot 383<\cosh \left\{a\left(\frac{a-3}{a+1}\right)^{\frac{1}{2}}\right\} \leqslant \cosh \left\{a\left(\frac{a-3}{a-3+2 s}\right)^{\frac{1}{2}}\right\}$.

(ii) By Lemma 9 (i) we have to show that

$$
\operatorname{ar}\left\{1-\frac{1}{a^{2}}\left[\cosh ^{-1}(\zeta(2 s-1))\right]^{2}\right\} \geqslant 2 s
$$

for $r=3,4,5, \ldots$, when $1 \cdot 035 \leqslant s \leqslant 2$, i.e. that

$$
\zeta(2 s-1) \leqslant \cosh \left\{a\left(a-\frac{2}{3} s\right)\right\}^{\frac{1}{2}} \text {. }
$$

If $\frac{3}{2} \leqslant s \leqslant 2, \zeta(2 s-1) \leqslant \zeta(2)<\cosh \left\{a\left(a-\frac{4}{3}\right)\right\}^{\frac{1}{2}} \leqslant \cosh \left\{a\left(a-\frac{2}{3} s\right)\right\}^{\frac{1}{2}}$. Hence we need only prove that, if $1 \cdot 035 \leqslant s \leqslant \frac{3}{2}$ then

Now, if $\mathrm{I}<s \leqslant \frac{3}{2}$,

$$
g(s)=\log \cosh \left\{a\left(a-\frac{2}{3} s\right)\right\}^{\frac{1}{2}}-\log \zeta(2 s-1) \geqslant 0 .
$$

$$
\begin{aligned}
g^{\prime}(s) & =-\frac{1}{3}\left\{\frac{a}{a-\frac{2}{3} s}\right\}^{\frac{1}{2}} \tanh \left\{a\left(a-\frac{2}{3} s\right)\right\}^{\frac{1}{2}}-2 \frac{\zeta^{\prime}}{\zeta}(2 s-1) \\
& >-\frac{1}{3}\left(\frac{a}{a-1}\right)^{\frac{1}{2}} \tanh \left\{a\left(a-\frac{2}{3}\right)\right\}^{\frac{1}{2}}-2 \frac{\zeta^{\prime}}{\zeta}(2)>0 \cdot 751>0,
\end{aligned}
$$

since $\frac{\zeta^{\prime}}{\zeta}(2)=-0.5699610$. Hence $g(s)$ is an increasing function for $1<s \leqslant \frac{3}{2}$, and since $g\left(\frac{3}{2}\right)>0$ it follows that there exists a unique $s_{0}$ such that $1<s_{0}<\frac{3}{2}$ and $g\left(s_{0}\right)=0$. By using Gram's tables of $(s-1) \zeta(s)$ we find that $1.03<s_{0}<1.035$, and this completes the proof.

(iii) By Lemma 9 (i) we have to show that

$$
\zeta(2 s-1) \leqslant \cosh \{4 \pi(\pi-s)\}^{\frac{1}{2}}
$$

for $2 \leqslant s \leqslant 3$. This is true since $\zeta(3)<\cosh \{4 \pi(\pi-3)\}^{\frac{1}{2}}$.

3. We now prove the Theorem for $s \geqslant 3$.

Suppose first that $\alpha+\beta+\gamma \leqslant 4$. Then we have, by (9) and Lemma 3,

$$
\begin{aligned}
Z_{h}(s) & =\frac{1}{3}\left\{Z_{f}(s)+Z_{g}(s)+Z_{h}(s)\right\} \\
& =\frac{1}{3} \sum_{m} \sum_{n}^{\prime}\left\{f^{-s}+g^{-s}+h^{-s}\right\} \\
& \geqslant\left(\frac{1}{2} \sqrt{ } 3\right)^{s} \sum_{m} \sum_{n}^{\prime} q^{-s}=Z_{Q}(s),
\end{aligned}
$$

equality occurring only when $h=Q$. To complete the proof we have therefore to consider the case $\alpha+\beta+\gamma \geqslant 4$. Now we have, by (14) and (15)

$$
Z_{h}(s)=y^{s} \sum_{m} \Sigma_{n}|m z+n|^{-2 s}>2 \zeta(2 s) \phi(x, y ; s),
$$

since the last expression is the part of the double sum corresponding to the terms

for $r=1,2,3, \ldots$.

$$
(m, n)=(0, \pm r), \quad( \pm r, 0), \quad( \pm r, \mp r)
$$


By Lemma 4 and (4) it remains to prove that

where

$$
F(s)=2+2^{-s}-3\left(\frac{1}{2} \sqrt{ } 3\right)^{s} \psi(s) \geqslant 0,
$$

$$
\psi(s)=\frac{\zeta(s) L(s)}{\zeta(2 s)}=\left(1+3^{-s}\right) \underset{p \equiv 1(\bmod 3)}{\prod_{2}}\left(\frac{1+p^{-s}}{1-p^{-s}}\right) .
$$

Now it is easily shown by the method described in the appendix to a paper by D. G. Kendall $\dagger$ that $L(3)=0.8840238$ and so $F(3)>0.0896>0$. Also, by considering the infinite product, we see that $\psi(s)-1$ is a decreasing function of $s$, and since $2^{-s}-3\left(\frac{1}{2} \sqrt{ } / 3\right)^{s}$ is an increasing function it follows that $F(s)$ is an increasing function and is therefore positive.

4. In this section we assume that

$$
1 \cdot 035 \leqslant s \leqslant 3 .
$$

The function $G(x, y)$ of $(15)$ is an even periodic function of $x$ of period unity ; we express it as a Fourier series :

We have, for $r>0$,

$$
G(x, y) \sim \sum_{r=-\infty}^{\infty} a_{r} e^{2 \pi i r x}=a_{0}+2 \sum_{r=1}^{\infty} a_{r} \cos 2 \pi r x
$$

$$
a_{r}=\int_{-\frac{1}{2}}^{t} G(x, y) e^{-2 \pi i r x} d x=2 y^{s} \sum_{m=1}^{\infty} \sum_{n=-\infty}^{\infty} \int_{-\frac{1}{2}}^{\frac{1}{2}} \frac{e^{-2 \pi i r x} d x}{\left\{(m x+n)^{2}+m^{2} y^{2}\right\}^{s}} .
$$

In the inner sum we put $n=m \lambda+\nu$ where $0 \leqslant \nu<m$ and obtain

$$
\begin{aligned}
a_{r} & =2 y^{s} \sum_{m=1}^{\infty} \sum_{\nu=0}^{m-1} \int_{-\infty}^{\infty} \frac{e^{-2 \pi i r x} d x}{\left\{(m x+\nu)^{2}+m^{2} y^{2}\right\}^{s}} \\
& =2 y^{s} \int_{-\infty}^{\infty} \frac{e^{-2 \pi i r t} d t}{\left(t^{2}+y^{2}\right)^{s}} \sum_{m=1}^{\infty} \frac{1}{m^{2 s}} \sum_{\nu=0}^{m-1} e^{2 \pi i r v / m} \\
& =2 y^{s} \int_{-\infty}^{\infty} \frac{e^{-2 \pi i r t} d t}{\left(t^{2}+y^{2}\right)^{s}} \sum_{m \mid r} m^{1-2 s} \\
& =\frac{4 \pi^{s} y^{\frac{1}{2}}}{\Gamma(s)} r^{s-\frac{1}{2}} \sigma_{1-2 s}(r) K_{s-\frac{1}{s}}(2 \pi r y),
\end{aligned}
$$

where

$$
\sigma_{k}(n)=\underset{d \mid n}{\sum} d^{k}
$$

Similarly it can be shown that

$$
a_{0}=2 y^{s} \zeta(2 s)+2 y^{1-s} \zeta(2 s-1) \frac{\Gamma\left(\frac{1}{2}\right) \Gamma\left(s-\frac{1}{2}\right)}{\Gamma(s)} .
$$

The inversions of the orders of summation are all justified because of the absolute convergence of the series concerned. Also the Fourier series is uniformly convergent in $x$, and so we have, in fact,

$$
G(x, y)=a_{0}+2 \sum_{r=1}^{\infty} a_{r} \cos 2 \pi r x
$$

Also, again by uniform convergence,

$$
\begin{aligned}
G_{x}(x, y) & =-4 \pi \sum_{r=1}^{\infty} r a_{r} \sin 2 \pi r x \\
& =-\frac{16 \sqrt{ } \pi}{2^{s+\frac{1}{2}} y^{s} \Gamma(s)} \sum_{r=1}^{\infty} \lambda_{r} \sin 2 \pi r x=-A \sum_{r=1}^{\infty} \lambda_{r} \sin 2 \pi r x,
\end{aligned}
$$

$\dagger$ " On the number of lattice points inside a random oval," Quart. J. Math., Oxford Ser. 19 (1948), $1-26$. 
say, where

$$
\lambda_{r}=\sigma_{1-2 s}(r) H(2 \pi r y),
$$

in the notation of (17). By partial summation we obtain, for $x \neq 0$,

$$
G_{x}(x, y)=-\frac{A}{4 \sin ^{2} \pi x} \sum_{r=1}^{\infty}\left(\lambda_{r+2}-2 \lambda_{r+1}+\lambda_{r}\right)\{(r+1) \sin 2 \pi x-\sin 2 \pi(r+1) x\} .
$$

Now since

$$
\begin{array}{ll}
1 \leqslant \sigma_{1-2 s}(r) \leqslant 1+2^{1-2 s} & (r=1,2,3) \\
1 \leqslant \sigma_{1-2 s}(r)<\zeta(2 s-1) & (r \geqslant 1),
\end{array}
$$

it follows that we shall have

if

$$
\lambda_{r+2}-2 \lambda_{r+1}+\lambda_{r}>0 \quad(r=1,2,3, \ldots)
$$

$$
H\{(r+2) h\}-2 \cosh \mu_{r} h H\{(r+1) h\}+H(r h)>0 \quad(r=1,2, \ldots),
$$

where $h=2 \pi y$ and $\mu_{r}$ is defined by

$$
\begin{array}{lr}
\cosh \mu_{r} h=1+2^{1-2 s} & (r=1,2 ; s \leqslant 2), \\
\cosh \mu_{r} h=\zeta(2 s-1) & \text { (otherwise). }
\end{array}
$$

By Lemmas 8 and 10 with $u=r h$ we conclude that (21) holds in the following cases: (a) $1 \cdot 035 \leqslant s \leqslant 2, y \geqslant \frac{3}{5},(b) 2 \leqslant s \leqslant 3, y \geqslant 1$. It follows from $(20)$ that $G_{x}(x, y)<0$ in cases $(a)$ and $(b)$ if $0<x<\frac{1}{2}$.

5. We now suppose that $2<s \leqslant 3$. By Lemma 7, the minimum of $G(x, y)$ is attained at a point $z$ of $z$ for which $y \leqslant \frac{3}{2}$. Now $z$ must lie in the circle $\left(x-\frac{1}{2}\right)^{2}+(y-1)^{2} \leqslant \frac{1}{4}$ as otherwise $y \geqslant 1$, and, by case $(b)$ of $\S 4$, it would follow that $G(x, y)$ could be diminished by increasing $x$. Hence $\left(x-\frac{1}{2}\right)^{2}+(y-1)^{2} \leqslant \frac{1}{4}$, i.e. $\alpha+\beta+\gamma \leqslant 4$, and the argument given at the beginning of $\S 3$ shows that $G(x, y) \geqslant G\left(\frac{1}{2}, \frac{1}{2} \sqrt{ } 3\right)$, equality occurring only when $x=\frac{1}{2}, y=\frac{1}{2} \sqrt{ } 3$.

6 . It remains to consider the range $1 \cdot 035 \leqslant s \leqslant 2$. Again by Lemma 7 we know that $G(x, y)$ attains its minimum at a point $z$ of $\mathbb{Z}$ for which $y \leqslant \frac{3}{2}$. Since $G_{x}(x, y)<0$ in except on $x=0$ and $x=\frac{1}{2}$ it follows that $z=\frac{1}{2}+i y$ where $\frac{1}{2} \sqrt{ } 3 \leqslant y \leqslant \frac{3}{2}$. Now, if $\frac{1}{2} \sqrt{ } 3<y \leqslant \frac{3}{2}, G(x, y)$ takes the same value at the point

$$
z^{\prime}=x^{\prime}+i y^{\prime}=-\frac{1}{z-1}=\frac{2}{4 y^{2}+1}+i \frac{4 y}{4 y^{2}+1} .
$$

But $0<x^{\prime}<\frac{1}{2}$ and $y^{\prime} \geqslant \frac{3}{5}$ so that $G_{x}\left(x^{\prime}, y^{\prime}\right)<0$ and hence $G(x, y)$ can be decreased still further by increasing $x^{\prime}$. This contradiction establishes that $Z_{h}(s)$ attains its minimum at $z=\frac{1}{2}+i_{2}^{1} \sqrt{ } 3$ and at no other point of $\mathbb{7}$; i.e. the minimum is attained only when $h(m, n)=Q(m, n)$. This completes the proof of the theorem.

We remark, in conclusion, that it is of course possible to reduce the lower bound 1.035 of $s$ somewhat by more detailed arithmetical analysis, but, since the method places an upper bound on $\zeta(2 s-1)$, it cannot be used without alteration to prove the Theorem for all $s>1$.

The University, Birmingham, 15 